\title{
Histomorphometric Study of Neuroglial Elements of Trigeminal Ganglion in Young Adult and Aged Animals Following Tooth Extraction
}

\author{
Shahriar Ahmadpour ${ }^{1}$, Arman Behrad ${ }^{2}$, Khadijeh Foghi ${ }^{3}$, Luis Rafael Moscte-Salazar ${ }^{4}$
}

\begin{abstract}
Trigeminal ganglion (TG) is the main sensory ganglion of the orofacial regions associated with neuropathic pain. TG comprises pseudo-unipolar neurons with different morphologies and two types of glial cells, including Schwann cells and satellite glial cells (SGC). We designed this study to examine the effects of tooth extraction (as a cause of neuropathic pain) on the morphological and population change in SGC and neurons of the TG in aged and young adult animals. The results of our current study revealed that tooth extraction is not associated with quantitative changes in the number of SGC and leads to morphological changes in heterogeneous SGC of the ipsilateral side. The hypertrophic SGC was the most striking microscopic feature of the study. Additionally, quantitative changes in the pattern distribution and morphometry of neurons and the dynamic nature of the TG after tooth extraction particularly in aging was the other finding of our study.

Keywords: Neuroglial, Neuropathy, Tooth extraction, Trigeminal ganglion.

International Journal of Experimental Dental Science (2020): 10.5005/jp-journals-10029-1206
\end{abstract}

\section{INTRODUCTION}

Trigeminal ganglion (TG) is the main sensory ganglion of the orofacial regions. It comprises pseudo-unipolar neurons with different morphologies from the ophthalmic, maxillary and mandibular divisions and two types of glial cells, including Schwann cells and satellite glial cells (SGC). ${ }^{1}$ The diversity in the morphology and size of the TG neurons have been suggested to be involved in pain and proprioceptive modalities. ${ }^{2,3}$ Studies have revealed SGCs directly modulate neuronal homeostasis. ${ }^{4}$ Additionally, SGCs have been shown to be involved in neuropathic inflammation. ${ }^{5}$ Neuropathic pain caused by peripheral nerve injury is a common experience after tooth extraction. ${ }^{6}$ Neuropathic study has shown that tooth extraction is associated with increased number of GFAP-positive SGCs. ${ }^{2}$ Furthermore, cognitive impairment and astrogliosis have been reported after tooth extraction. ${ }^{7}$ SGCs express GFAP and envelope neurons and are considered as astrocytes-related cells outside the central nervous system. The dynamic interaction between the SGCs and the TG neurons seemed to play an important role in the normal physiologic and pathophysiology of the TG-related disorders such as migraine and trigeminal neuralgia. ${ }^{3}$

On the other hand, studies have documented evidence indicating tooth loss and aging process increase the risk of neurodegenerative disorders such as Alzheimer's disease and dementia. ${ }^{8}$ Aging is associated with various extensive changes in the central and peripheral nervous systems. Interestingly, it has been shown that aging process is associated with the changes in astroglial reactivity. ${ }^{9}$ However, there is little information about the histomorphometric changes in the neuroglial elements of TG in aging. Therefore, this study was designed to examine the effects of tooth extraction on the SGC and neuronal population of the TG in aged and young adult animals. ${ }^{1-3}$ Department of Anatomy, North Khorasan University of Medical
Sciences, Bojnurd, North Khorasan, Islamic Republic of Iran
${ }^{4}$ Health Sciences and Neurosciences Research Group (CISNEURO),
Cartagena de Indias, Colombia

Corresponding Author: Arman Behrad, Department of Anatomy, North Khorasan University of Medical Sciences, Bojnurd, North Khorasan, Islamic Republic of Iran, Phone: +989389455310, e-mail: arman.behi@gmail.com

How to cite this article: Ahmadpour S, Behrad A, Foghi K, et al. Histomorphometric Study of Neuroglial Elements of Trigeminal Ganglion in Young Adult and Aged Animals Following Tooth Extraction. Int J Experiment Dent Sci 2020;9(2):43-46.

Source of support: Nil

Conflict of interest: None

\section{Materials and Methods}

All protocols and experiments were approved by the ethics committee of North Khorasan University of Medical Sciences (NKUMS). Twenty male Wistar rats were divided into groups, namely, young adults' control (6 weeks old, weighing 150-200 g), young adults (experiment) old control, and old experiment groups (36 weeks old, weighing 200-250 g). The animals were acclimatized for 1 week before surgical experiment. The rats were anesthetized by ketamine $(70 \mathrm{mg} / \mathrm{kg})$ and chlorpromazine. ${ }^{10}$ Under deep intraperitoneal anesthesia, the upper right incisor tooth was extracted using dental forceps according to the previous studies. ${ }^{3}$ The sockets were packed with animals' antibiotic. After recovery, the rats were fed by powered food. Seven days after extraction, the animals were deeply anesthetized by chloroform and transcardially perfusion were performed with 
formalin and saline. The right and left TGs were removed carefully and post-fixed in $10 \%$ formalin. The samples were cut into $10 \mu \mathrm{m}$ slices and selected according to systematic random sampling. The selected sections were stained with HE. ${ }^{11}$ From each animal,10 selected fields were studied and captured. Quantification of the parameters, including the diameter of neurons and counting of SGC, were performed with Cellsens software. For microscopic examination, each section was divided into three concentric zones (Fig. 1). The measured diameters of the neurons were divided into three sizes using SPSS software. ${ }^{1}$

\section{Statistical Analysis}

The obtained results were expressed as mean \pm SD. Statistical analyzes were conducted by Statistical Product for Social Sciences (SPSS version 17.0), one-way Kruskal-Wallis, and independent $t$ test. Differences were considered to be significant at $p$ value $<0.05$.

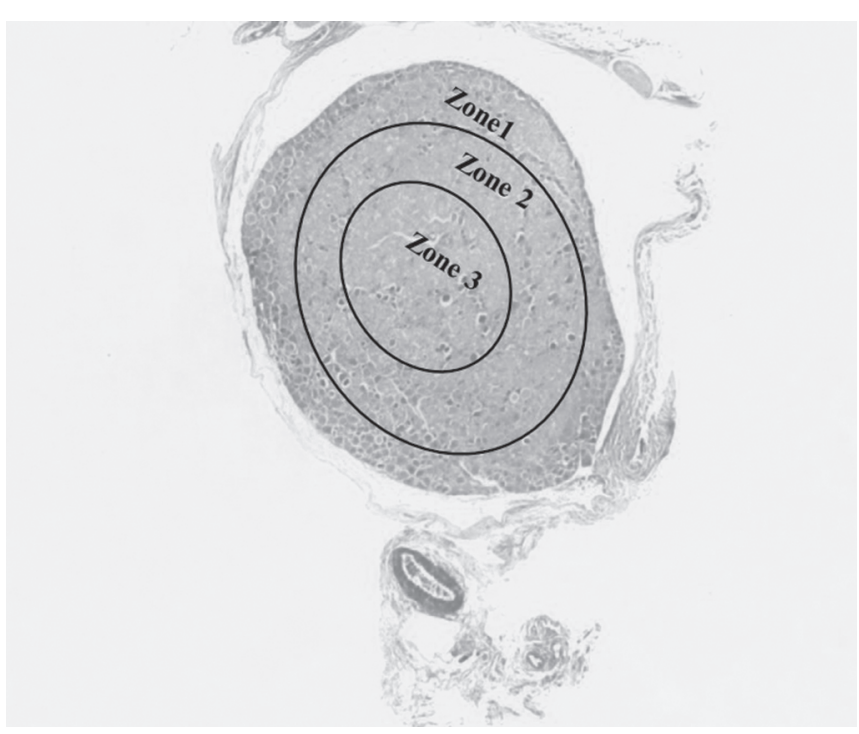

Fig. 1: Manner of dividing each section for microscopic examination

\section{Results \\ Quantitative Findings}

- The mean of SGC number in the right TG of young animals (24:80 $\mu \mathrm{m} \pm 5: 11 \mu \mathrm{m})$ and the left side $(11: 20 \mu \mathrm{m} \pm 6: 90 \mu \mathrm{m})$ showed no significant level of difference ( $p$ value $=0.051$ ). The comparison between the number of SGC in the right TG of aged group (14:40 $\mu \mathrm{m} \pm 5: 12 \mu \mathrm{m})$ and those of the left TG (11:20 $\pm 6: 90)$ showed no meaningful difference ( $p$ value $=0.915$ ). Significant difference was found in comparison between the right young TG and the left old TG ( $p$ value $=0.016$ ).

- The mean size of the neurons in the right TG of young animals $(16: 91 \mu \mathrm{m} \pm 4: 84 \mu \mathrm{m})$ and the left side $(13: 89 \mu \mathrm{m} \pm 4: 27 \mu \mathrm{m})$ showed no meaningful difference $(p<0.05)$.

- The neurons of the TG were classified into less than $18 \mu \mathrm{m}(C)$, between 18 and $22 \mu \mathrm{m}$ (B), and more than $22 \mu \mathrm{m}$ (A) (Fig. 2).

\section{Descriptive Findings}

Neurons

Young group: Extracted group: Compacted layers of the large neurons in the periphery and central zones of the right TG were seen, while polygonal and acidophil neurons were found in the central zones of the right TG. In the left TG, large neurons were seen in one quadrant and scattered polygonal neurons were observed (Fig. 3).

Control group: In the right side, in the peripheral region of TG, multiple columns of large neurons with round and polygonal shape were observed. In the left side, these shapes were not found in the peripheral and central areas.

Old group: Extracted group: Circular and large neurons were seen in the periphery of the right TG, while polygonal types were found in the deeper zones.

Control group: In the right side, two columns of large neurons with round and polygonal shape were observed in the peripheral region of TG. Polygonal types were found in the deeper zones.

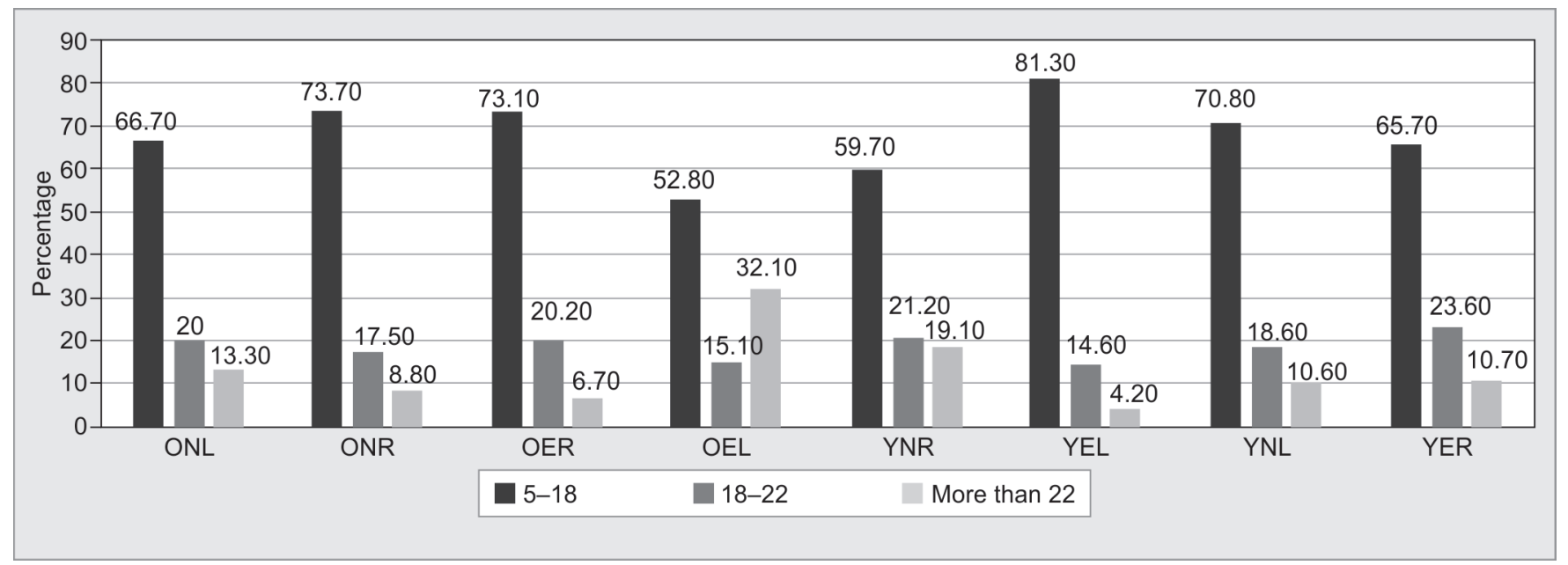

Fig. 2: Quantity of different groups of neurons in right and left TG in different group 


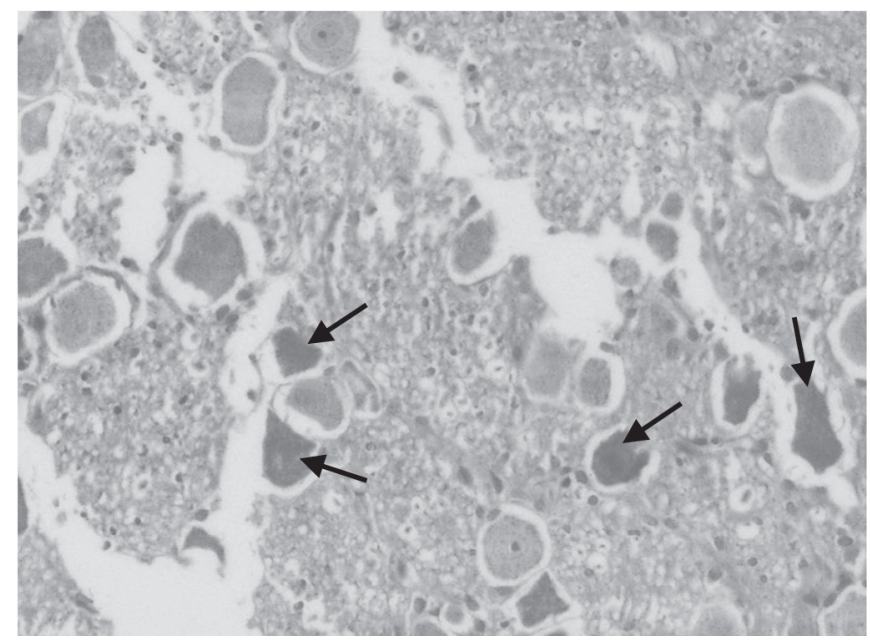

Fig. 3: Microscopic examination of Young group right TG after extraction: large neurons and scattered polygonal neurons mostly in central areas X400

\section{SGC}

Young group: Extracted group: After extraction, in the right TG round, hypertrophic SGCs were observed surrounding the large dark polygonal neurons (Fig. 4). On the left side, only round dark SGCs were observed in perineuronal regions.

Control group: In the control group, dark round SGCs were found in the interneuronal matrix of TG. Some light large SGCs were also found in perineuronal and perivascular regions in both side TG.

Old group: Extracted group: Tooth extraction induced different types of SGCs, including dark round and elongated satellite glial cells, which were found on the right side. There were seen some light hypertrophic SGCs among the large polygonal neurons on both sides.

Control group: Round dark SGCs in interneuronal regions of the right TG were observed. On the left side scattered large light rounded SGCs were found between the polygonal types of neurons.

\section{Discussion}

The results of our current study revealed that tooth extraction (as a peripheral trauma) is not associated with quantitative changes in the number of SGC and leads to morphological changes in heterogeneous SGC of the ipsilateral side. Additionally, our findings showed the hypertrophic SGC as the most striking microscopic feature after tooth extraction. The SGCs are a group of heterogeneous and pleomorphic glial cells that are involved in various functions including neurotransmitter regulation, potassium uptake, energy metabolism regulation, and blood-brain barrier. ${ }^{12}$ Altered function of satellite glial cells has been speculated in the neuroinflammatory and neurodegenerative disorders. ${ }^{9}$

Experimental studies have demonstrated that SGCs respond to the external stimulation with increases in the intracellular $\mathrm{Ca}^{2+}$ level and transmit these calcium signals to adjacent nonstimulated SGCs as intracellular $\mathrm{Ca}^{2+}$ waves, increasing TG neuron excitability. ${ }^{13}$ But the role of SGCs in neuropathic pain that resulted from tooth extraction has not been thoroughly understood. The study has also shown glial cells are involved in the regulation of inflammation and pain processes. ${ }^{14}$ In this study, microscopic examination

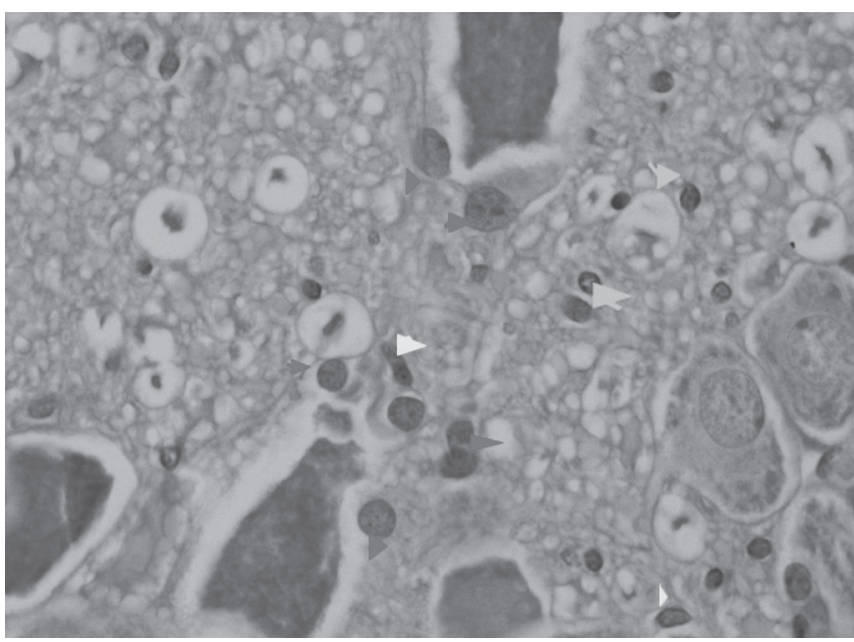

Fig. 4: Microscopic examination of Young group right TG after extraction: hypertrophic SGCs (red arrows head) SGCs with bright and elongated nuclei (yellow arrows head) and astrocytes with more compacted and round nuclei (blue arrows) X1,000

revealed reactive hypertrophic SGCs which are only found in the ipsilateral ganglion of the young experimental animals. Such reactive SGCs indicate the increased neuroglial interaction during the inflammatory processes. Previous studies could also provide evidence indicating the morphologic changes in SGCs and also increased level of GFAP protein in the hippocampus after tooth extraction. ${ }^{15}$ Although due to some technical limitation we could not take advantage of the immunohistochemistry, our results are in line with Onozuka et al. ${ }^{16} \mathrm{He}$ showed gliosis and hypertrophic SGC after tooth extraction.

Another interesting finding of this study was related to hypertrophic SGCs. These cells were only found in young experimental animals. The different reactions of SGCs in response tooth extraction may be residing in the age-related disturbance in the function of SGCs, the difference in the time of the cellular reaction to the inflammatory factors, and the level of the neuropathic pain processing. ${ }^{9,17}$ Furthermore, our findings revealed that tooth extraction is associated with the quantitative changes in the pattern distribution and morphometry of neurons in TG. The frequency of neural size showed a different pattern in the young and old experiment groups.

The comparison between the frequency of neuronal size in young and old experimental groups revealed that tooth extraction may lead to a neuronal shift in the contralateral side TG. Precisely, the old experimental shift to $A$ on the left side while in the young experiment shift to $C$ on the left side was noticed.

According to the recent findings, each type of neuronal population in the TG is involved in a specific sensory modality. ${ }^{18}$ For instance, small neurons are involved in pain conduction, and larger ones mediate other stimuli. Interestingly, the mean size of the neurons in the left TG of the young experiment group showed reduction while in the old experiment group the mean size of neurons in the bilateral TG showed no significant changes. Meaningful lateralization after tooth extraction concerning neuronal shift signifies the plastic nature of the neuronal population of the TG in which some intermediary types of neurons substitute other types in response to the peripheral stimuli. ${ }^{19,20}$

Given the functional differences between the diverse neuronal types in the TG, such neuronal shift after tooth extraction could 
propose as an adaptation to preserve and balance the behavioral homeostasis. Therefore, more frequent small neurons in the young animals could be considered as an adaptation to pain and exteroceptive modalities, while in contrast, the old animals' response differently as a shift to large neurons in adaptation maintain the proprioceptive modalities.

In summary, our study could provide evidence regarding the dynamic nature of the TG after tooth extraction particularly in aging.

\section{Conclusion}

Tooth extraction (as a peripheral trauma) is not associated with quantitative changes in the number of SGC and leads to morphological changes in heterogeneous SGC of the ipsilateral side. These pathological changes could propose as an adaptation to preserve and balance the behavioral homeostasis. Interestingly, different mechanisms of adaption experienced between young and old groups; therefore, we suggest a comprehensive study to evaluate the effects of the TG alteration in pain and proprioceptive modalities in a different group of ages.

\section{Acknowledgments}

All authors participated in the design of this study, experimental, and pathological studies. All authors have read and approved the content of the manuscript.

\section{References}

1. Lagares $A$, Avendaño C. Lateral asymmetries in the trigeminal ganglion of the male rat. Brain Res 2000;865(2):202-210.

2. Pk S, Al Dajah SRSB. Morphological study of nociceptive neurons in the trigeminal ganglion. Int J Health Rehab Sci 2017;5(1):1-10.

3. Gunjigake KK, Goto T, Nakao K, et al. Activation of satellite glial cells in rat trigeminal ganglion after upper molar extraction. Acta Histochem Cytochem 2009;42(5):143-149.

4. Costa FAL, Neto FLM. Satellite glial cells in sensory ganglia: its role in pain. Rev Bras Anestesiol 2015;65(1):73-81.

5. Ohara PT, Vit JP, Bhargava A, et al. Gliopathic pain: when satellite glial cells go bad. Neuroscientist 2009;15(5):450-463.
6. Tinastepe N, Oral K. Neuropathic pain after dental treatment. Agri 2013;25(1):1-6.

7. Kubo K-y, Murabayashi C, Kotachi M, et al. Tooth loss early in life suppresses neurogenesis and synaptophysin expression in the hippocampus and impairs learning in mice. Arch Oral Biol 2017;74: 21-27.

8. Dioguardi M, Di Gioia G, Caloro GA, et al. The association between tooth loss and Alzheimer's disease: a systematic review with metaanalysis of case control studies. Dent J (Basel) 2019;7(2):49.

9. Palmer AL, Ousman SS. Astrocytes and aging. Front Aging Neurosci 2018;10:337.

10. Ahmadpour S, Foghi K, Behrad A. Chronic exposure to ketamine induces neuronal lose and glial reaction in CA4 region of hippocampus. J Morphol Sci 2016;33(2):103-107.

11. Bancroft J, Stevens A, Tumer D. Theory and practice of histological techniques. 3rd ed., Edinburgh: Churchil Livinsgstone; 1990. pp. 360-361.

12. Verkhratsky A, Nedergaard M. Physiology of astroglia. Physiol Rev 2018;98(1):239-389.

13. Gu Y, Chen $Y$, Zhang $X$, et al. Neuronal soma-satellite glial cell interactions in sensory ganglia and the participation of purinergic receptors. Neuron Glia Biol 2010;6(1):53-62.

14. Berge TI. Incidence of chronic neuropathic pain subsequent to surgical removal of impacted third molars. Acta Odontol Scand 2002;60(2):108-112.

15. linuma $\mathrm{M}$, Kondo $\mathrm{H}$, Kurahashi $\mathrm{M}$, et al. Relationship between the early toothless condition and hippocampal functional morphology. Anat Physiol 2014;4(3):8-13.

16. Onozuka $M$, Watanabe $K$, Fujita $M$, et al. Evidence for involvement of glucocorticoid response in the hippocampal changes in aged molarless SAMP8 mice. Behav Brain Res 2002;131(1-2):125-129.

17. Hatashita S, Sekiguchi M, Kobayashi H, et al. Contralateral neuropathic pain and neuropathology in dorsal root ganglion and spinal cord following hemilateral nerve injury in rats. Spine 2008;33(12): 1344-1351.

18. Bear MF, Connors BW, Paradiso MA. Neuroscience. Filadelfia: Lippincott Williams and Wilkins; 2007.

19. Lynds R, Lyu C, Lyu GW, et al. Neuronal plasticity of trigeminal ganglia in mice following nerve injury. J Pain Res 2017;10:349.

20. Jacquin MF, Rhoades RW. Development and plasticity in hamster trigeminal primary afferent projections. Dev Brain Res 1987;31(2): 161-175. 